\title{
L'arrivée du chemin de fer à Brest ou les effets d'une logique stratégique
}

When railway lines reached Brest, or the effects of strategic planning

Christophe Le Bollan

\section{OpenEdition}

\section{Journals}

Édition électronique

URL : https://journals.openedition.org/rhcf/420

DOI : $10.4000 /$ rhcf.420

\section{Éditeur}

Rails \& histoire

Édition imprimée

Date de publication : 31 mai 2008

Pagination : $72-86$

ISSN : 0996-9403

Référence électronique

Christophe Le Bollan, «L'arrivée du chemin de fer à Brest ou les effets d'une logique stratégique »,

Revue d'histoire des chemins de fer [En ligne], 38 | 2008, mis en ligne le 10 mai 2011, consulté le 22 avril 2022. URL : http://journals.openedition.org/rhcf/420 ; DOI : https://doi.org/10.4000/rhcf.420 


\title{
L'arrivée du chemin de fer à Brest ou les effets $d^{\prime}$ une logique stratégique
}

\author{
Christophe LE BOLLAN
}

n cette première moitié du XIX e siècle, la France se remet
tranquillement des soubresauts de la Révolution et de l'Em-
pire et amorce, sous la monarchie de Juillet, un processus de modernisation industrielle. Par la loi du 11 juin 1842, elle entérine en particulier la création d'un réseau ferroviaire national qui, par sa disposition en étoile autour de Paris, est appelé à générer ici et là des initiatives. Partout, un vent nouveau souffle et la ville de Brest ne tarde pas elle aussi à être bientôt attirée par les sirènes du progrès. L'arrivée éventuelle du chemin de fer dans la cité du Ponant laisse clairement entrevoir une possibilité d'émancipation urbaine pour un ensemble qui, depuis le XVII siècle, occupe une fonction particulière dans la défense de la France ${ }^{1}$. Mais, recroquevillée sur elle-même, cette fière sentinelle maritime n'a guère de perspectives d'évolution spatiale et conserve plus que jamais sa dualité urbaine : Brest, anticlérical et libéral, s'oppose à Recouvrance, quartier bretonnant. Ce constat n'échappe pas à la récente municipalité ${ }^{2}$ qui, créée par l'ordonnance royale du 2 juillet 1839, souhaite bouleverser la donne. Les choses, pourtant, sont loin d'être simples. Les contingences stratégiques pèsent de tout leur poids et le Génie, responsable de la sécurité des installations, se révèle un censeur implacable

1- Richelieu, Colbert et Vauban vont progressivement en faire une place de premier ordre structurée par un port de guerre, un arsenal et une enceinte fortifiée.

2- Cette première municipalité est composée d'un maire et de trois adjoints, dont un pour Recouvrance. 
lorsqu'il s'agit d'évoquer une possible restructuration de la ville. En outre, dans les conversations de salon, les autorités militaires se montrent plutôt hostiles à toute desserte ferroviaire, se réfugiant derrière l'argument selon lequel la ville est conçue pour se satisfaire à elle-même et doit ainsi pouvoir s'opposer à des attaques venant tant du large que des terres. Le chemin de fer serait ainsi, selon elles, de nature à remettre en cause la cohérence d'un système défensif éprouvé depuis deux siècles. Quels projets concrets, malgré son inexpérience, la municipalité peut-elle alors soumettre sans écarter pour autant l'importance stratégique de la ville? Les militaires, de leur côté, peuvent-ils faire preuve d'ouverture ? Telle est la nature du débat qui se présente à Brest à la veille de la Seconde République et du Second Empire.

\section{La gare et le port ou la vision d'un projet global}

Malgré de louables intentions, l'arrivée du chemin de fer à Brest n'est pourtant pas initialement programmée. La «Charte» ferroviaire de juin 1842 ne prévoit en effet pour la péninsule bretonne qu'une ligne Paris-Nantes. Une prolongation vers Brest demeure encore très incertaine et les autorités administratives, comme c'est de coutume lorsqu'il s'agit de travaux publics, préfèrent procéder par étapes. En 1844, le principe d'une ligne complémentaire Paris-Rennes est néanmoins admis, laissant ainsi présager de belles perspectives pour Brest. Tout s'enchaîne alors et par l'entremise du Second Empire, qui met à l'étude en 1853 les contours d'un futur réseau breton, la liaison entre Rennes et Brest devient une chose convenue.

Alors que le tracé final vers Brest donne encore lieu à quelques tergiversations ${ }^{3}$, la municipalité en profite pour anticiper l'emplacement de la gare, laquelle est clairement perçue comme un pôle d'urbanisation. Depuis déjà plusieurs années, elle envisage en particulier de déplacer les fortifications vers les terres afin d'agrandir la ville et de répondre efficacement à la poussée démographique. Estimée à 37000 habitants en 1826, la population est en effet passée à 62000 âmes au milieu des années 1840 et il y a donc urgence. Les élus nourrissent en outre le secret espoir de pouvoir implanter la gare directement au sein de l'intramuros, dans la partie orientale de la ville, tout en sachant qu'une telle opération est de nature à compromettre les principes sécuritaires évoqués plus haut.

3- Le choix se portera finalement sur un tracé passant par l'anse de Kerhuon, adapté ainsi aux exigences de la zone frontière militaire imposée entre Landerneau et Brest. 
Mais rien n'indique que le Génie consentira à une telle concession. Dans l'immédiat, la municipalité s'applique à poser des conditions financières afin d'aboutir à un consensus. Elle est symboliquement soutenue en août 1855 par le conseil d'arrondissement qui, à l'unanimité, demande que l'agrandissement de Brest soit approuvé par le gouvernement. Consolidé dans ses principes et prêt à s'imposer de grands sacrifices, le conseil municipal vote ensuite, le 12 novembre, une subvention de 1,5 million de francs. Fort d'un enthousiasme sans faille, il surenchérit même une semaine plus tard, le 19 novembre, en proposant la création d'un nouveau port de commerce, présentée comme " étant la conséquence obligée de l'établissement du chemin de fer $»^{4}$, et pour laquelle il offre un subside d'un million de francs. Doit-on y voir un appel du pied à destination des militaires ? Peut-être, d'autant plus que les installations de la Royale, implantées sur la rivière Penfeld qui traverse la ville, sont peu fonctionnelles et doivent cohabiter avec les navires de commerce, eux aussi à l'étroit. C'est donc sur un projet global et ambitieux de modernisation de la ville que la municipalité s'engage 5 .

\section{L'interférence portuaire}

La Compagnie des chemins de fer de l'Ouest, s'accordant avec les vœux brestois, présente, dès le début de 1856, des propositions pour l'emplacement de la gare, afin que celui-ci puisse être combiné avec les rues prévues pour l'agrandissement de la ville. Le projet est soumis à l'enquête publique, laquelle se voit même attribuer, en raison du caractère non urgent de la situation locale, une durée d'un mois contre huit jours habituellement. Parallèlement, l'avant-projet de déplacement des fortifications, proposé et approuvé par les autorités militaires, progresse. L'opération s'élève au total à 6 millions de francs dont 1,5 million est couvert par la subvention municipale. Le 25 mai, le vicomte Aimé Le Coat de Kervéguen propose en outre au ministère de la Guerre d'acheter $120000 \mathrm{~m}^{2}$ de terrains occupés par les fortifications pour une somme de 2040000 F. De son côté, la Compagnie de l'Ouest consent à payer $1000000 \mathrm{~F}$ pour l'emplacement de la gare, ce qui fait un total de $4540000 \mathrm{~F}$, l'État n'ayant plus qu'à payer $1460000 \mathrm{~F}$. Le 9 juin suivant, le ministère de la Guerre précise qu'il n'y a pas de motifs

4- Lettre du ministre de la Guerre au comte de Kératry, 27 décembre 1869, Archives municipales de Brest, $2 \mathrm{H} 8.5$.

5- En complément, par décret du 26 avril 1856, la construction d'un pont tournant d'une portée de $117 \mathrm{~m}$ reliant Brest à Recouvrance est entérinée. Cet ouvrage, conçu par Cadiat et Oudry, est mis en service le 23 juin 1861 et mettra fin à l'utilisation de bacs tributaires des marées pour traverser la Penfeld. 
pour s'opposer du point de vue militaire à l'extension de l'enceinte, mais conditionne soudainement l'étude de la question à l'entérinement du projet de port de commerce. Or, à cette date, ce projet est encore officieux. La municipalité hésite alors, soulignant que le prélèvement immédiat de la somme de 2,5 millions de francs sur son budget s'avère difficile. Il faut, soit obtenir des crédits extraordinaires, soit ajourner indéfiniment le projet, ce qui l'amène finalement à retirer son offre pour la gare.

Comment alors interpréter ce changement soudain de politique ? Il apparait ici clairement que la municipalité a mal apprécié l'opération en cours. En dernier ressort, elle préfère abandonner l'extension de l'intra-muros pour investir dans le port de commerce dont l'utile construction doit stimuler l'activité marchande de la ville, jusqu'alors quasi inexistante. Le commerce se limite en effet à cette époque à la fabrication de toiles, de chapeaux et de chaux, tandis que les échanges maritimes sont peu développés, aspect qui est amplifié par l'archaïsme des installations de la Penfeld. Les échanges commerciaux concernent surtout la Marine, approvisionnée en houille par le Royaume-Uni et en bois par les pays nordiques, et le cabotage, notamment vers Landerneau. Brest occupe une place de moindre importance en comparaison, par exemple, du Havre qui enregistre, en 1846, un tonnage de jauge des navires de 360697 tonneaux contre 18617 tonneaux pour Brest ${ }^{6}$. Cet écart est révélateur du retard à combler et explique la mise à l'écart de la candidature brestoise le 20 février 1858 pour l'escale postale des paquebots transatlantiques, assurée par l'Union maritime, au profit de Cherbourg. Dans cet échec, l'absence de port de commerce et de desserte ferroviaire a indéniablement pesé.

En août 1858, Napoléon III, en visite en Bretagne, prend acte des vœux brestois et, par décret impérial du 24 août 1859, valide la création du Port-Napoléon à Porstrein, pour un coût global de 15 millions de francs, dont la contribution de la Ville. Une autre bonne nouvelle est enregistrée avec le désistement de l'Union maritime, qui se trouve finalement dans l'impossibilité de remplir ses obligations, en faveur de la Compagnie générale maritime et de la Société du crédit mobilier. Lors de l'examen du projet de loi devant ratifier une nouvelle convention, le comte de Tromelin, ancien député de Morlaix et membre de la commission chargée d'étudier la question, saisit l'occasion pour proposer Brest comme escale, à la place de Cherbourg. Cette modification

6- Yves Le Gallo (dir.), Histoire de Brest, Toulouse, Privat, 1976, p. 254.

7- Les sites de Landévennec et de Kerhuon avaient également été plébiscités. Ce dernier sera même un temps retenu mais, critiqué, il sera retiré. 
est adoptée par la commission, qui retient l'argument de la distance, Cherbourg se trouvant en effet plus éloigné de Brest de 115 milles. De cette manière, par un paquebot filant à 11 milles et demi à l'heure, voyageurs et dépêches en provenance des États-Unis peuvent débarquer au moins dix heures plus tôt à Brest; ce gain de temps est non négligeable, surtout pour les dépêches. La loi est votée en 1861 et Brest obtient l'escale postale des paquebots transatlantiques entre New York et Le Havre.

\section{Le choix final d'une gare isolée}

Avec la consolidation des intérêts portuaires, la question de la gare est reconsidérée. Celle-ci se voit alors attribuer un statut spécial, en rapport avec les exigences militaires. La meilleure solution technique consiste à la placer à environ $160 \mathrm{~m}$ des fortifications, sur le plateau est de la ville qui surplombe la rade entre le ravin dit du Gaz et le Petit Jardin, à $43 \mathrm{~m}$ au-dessus du niveau de la mer (fig. 1). L'emplacement, bordant la redoute de Kéroriou, est soumis à une nouvelle enquête du 29 octobre au 5 novembre 1860. Parmi les six observations consignées, une retient l'attention. Un groupement de propriétaires concernés par les terrains à exproprier suggère en effet de placer la gare sur les terrepleins du port. L'idée est pertinente dans la mesure où elle donne la possibilité de concentrer dans une gare maritime à la fois le trafic des marchandises et celui des voyageurs, et de réaliser notoirement une économie en évitant d'acheter des terrains pour l'implantation de la gare sur les hauteurs ${ }^{9}$. La commission d'enquête reconnait le bien-fondé de l'argument mais rétorque que cette disposition produirait de sérieux inconvénients. La gare, en effet, si elle était reportée sur le port, se trouverait à plus de $1250 \mathrm{~m}$ des portes de la ville et du faubourg de Paris, alors que, placée entre le ravin du Gaz et le Petit Jardin, elle est située à $500 \mathrm{~m}$ environ des portes, à $600 \mathrm{~m}$ du faubourg de Paris, et à $750 \mathrm{~m}$ du port. Cet emplacement aurait également pour conséquence d'isoler la vieille ville et « rendrait impossible le développement du centre de population, que l'existence même de la gare tendrait à produire. Il faudrait en effet prélever sur le terre-plein, qui est gagné à grande peine sur la rade, une superficie de dix hectares pour la gare, c'est-à-dire le tiers de la surface totale du terre-plein et diminuer d'autant l'espace sur lequel pourront être élevées les constructions indispensables au

8- Le ravin est ainsi dénommé en raison de la présence d'une usine à gaz en cet endroit. 9- L'achat des terrains sur le plateau révèle en effet un grand appétit des trente propriétaires expropriés puisque vingt-huit d'entre eux demanderont une indemnité globale de... $3407746 \mathrm{~F}$. L'attribution finale se soldera à $633000 \mathrm{~F}$. 
commerce $»^{10}$. Pour la commission, le problème du terre-plein peut être résolu en établissant, le long des quais, des voies ferrées en communication avec la voie prévue de raccordement du port. Finalement, le site proposé sur le plateau, d'une superficie totale de douze hectares, est maintenu par le ministère des Travaux publics le 10 août 1861. Ainsi, en plein accord avec les considérations stratégiques, les bâtiments de la gare sont reportés au-delà de la limite de la première zone des servitudes, tandis que la partie du périmètre de la gare qui pénètre dans la première zone des servitudes de la redoute de Kéroriou est comprise dans un polygone exceptionnel dans l'étendue duquel les constructions, de nature quelconque, peuvent être autorisées ${ }^{11}$.

Si cet emplacement ne permet pas d'offrir à la gare une destination quelque peu «monumentale » en rapport avec le contexte urbain, les élus s'évertuent encore à en rompre l'isolement supposé en suggérant de percer une voie directe à travers le front 22-24 de l'enceinte fortifiée, situé dans l'axe de la gare. Cette solution permettrait en outre de valoriser la gare en lui offrant un effet de perspective. Mais cette proposition est catégoriquement rejetée par le Génie qui, une fois de plus, invoque les intérêts défensifs. Il accepte néanmoins l'établissement d'une voie d'accès le long des fortifications, en pleine zone de servitude militaire, jusqu'à la porte de Landerneau, au nord-est de la ville. Conformément à l'article 13 du décret du 16 août 1853, il doit prendre en charge les travaux, mais ceux-ci, avec son accord et suite à la demande de l'ingénieur en chef Planchat ${ }^{12}$, sont intégrés dans une adjudication spéciale attribuée à l'entreprise Duchâteau ${ }^{13}$. Cette résolution permet ainsi de réaliser l'avenue, évaluée à $155000 \mathrm{~F}$, avec les fonds du chemin de fer, dont elle est la conséquence, en évitant l'emploi de l'entrepreneur général du Génie dont les prix, augmentés de $8 \%$, auraient été légèrement supérieurs à ceux du chemin de fer.

10- Rapport de la commission d'enquête, 23 novembre 1860, Archives départementales du Finistère, $5 \mathrm{~S} 36$.

11- Le chemin vicinal $n^{\circ} 8$ de Poullic-al-Lor au faubourg de Paris, coupé par la gare, est dévié de manière à passer par un passage inférieur établi sous le remblai du ravin de l'usine à gaz, et à se raccorder avec la direction existante, en faisant un lacet dans le coteau. Ce chemin, qui sert surtout au charroi des sables déchargés sur cette partie du rivage et que les cultivateurs viennent prendre pour l'amendement des terres, reste ainsi toujours en contact avec le littoral.

12- Louis-Henri Planchat (1837-1896) est chargé de la deuxième section de la ligne Rennes-Brest le $1^{\text {er }}$ juillet 1859, et de la ligne Landerneau-Châteaulin le 10 juillet 1861. Il entre ensuite à la direction de la Compagnie des chemins de fer de l'Ouest le $1^{\text {er }}$ août 1874 (Fichier Richard, École nationale des Ponts et Chaussées).

13- L'entreprise Duchâteau sera aussi chargée des terrassements de la gare. 


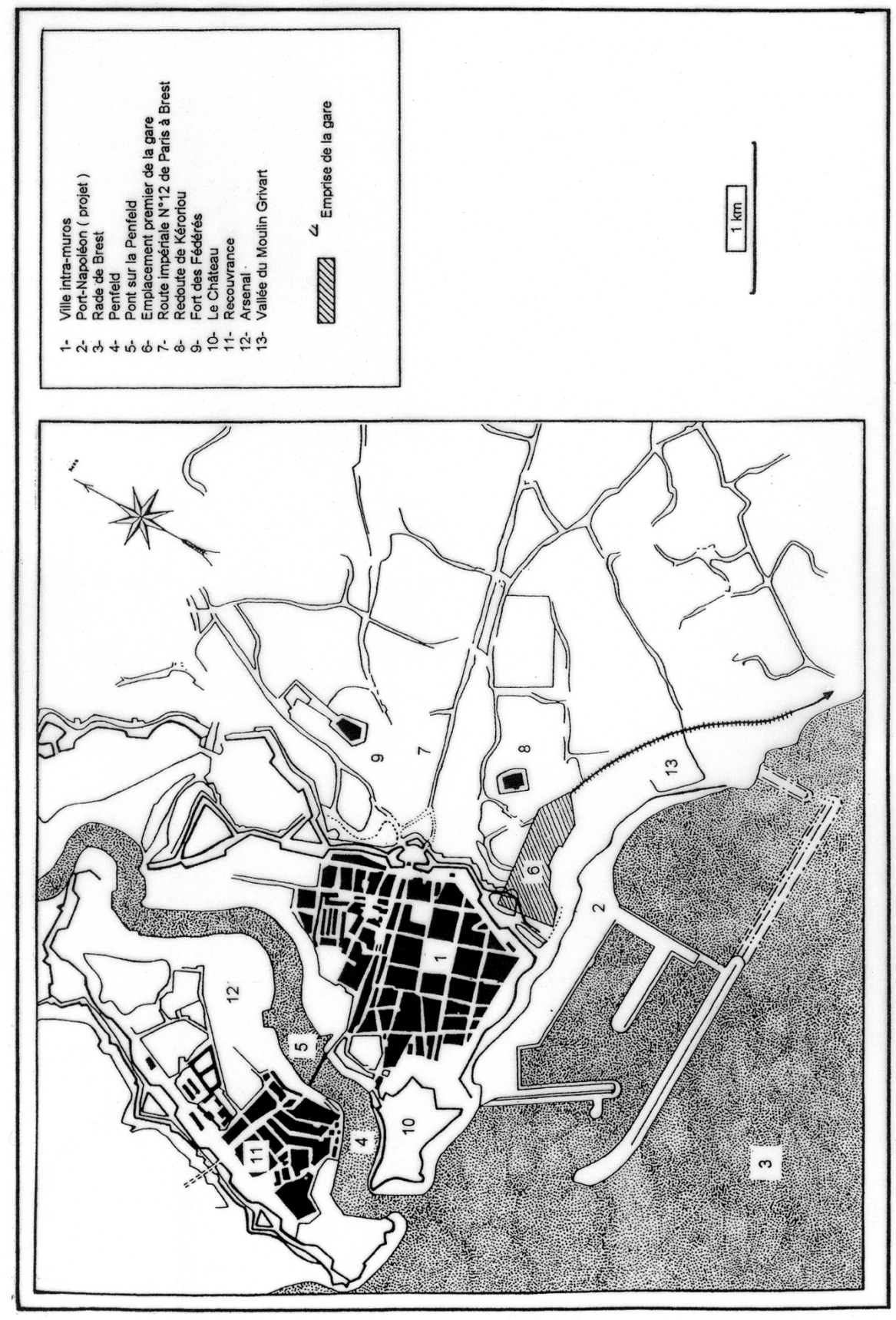

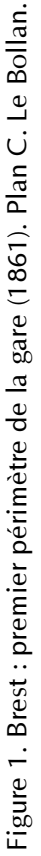




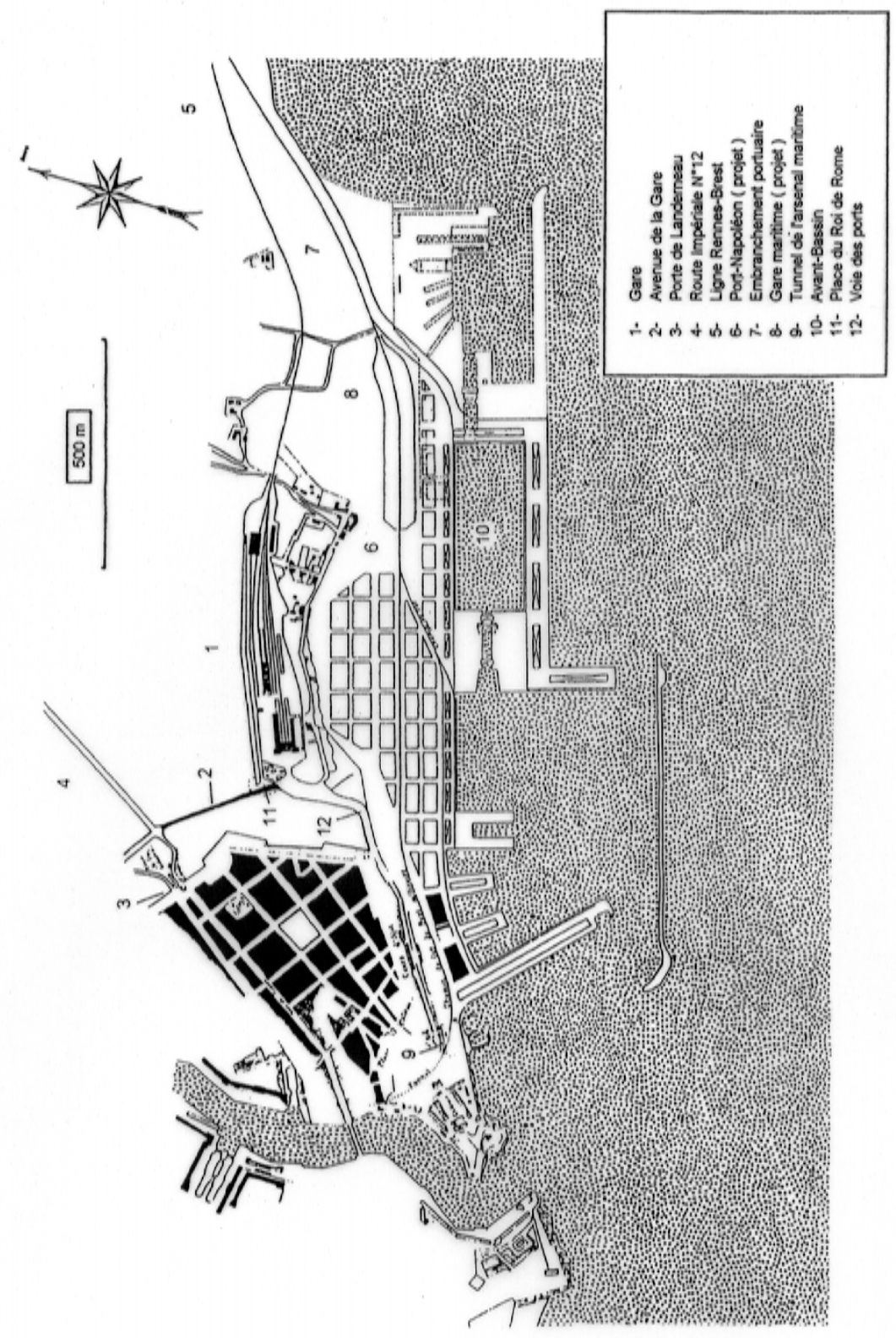

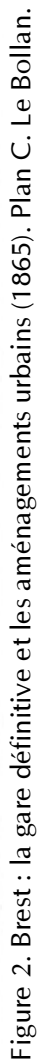


L'exécution de l'avenue, qui fournit $61000 \mathrm{~m}^{3}$ de déblais pour le port de commerce, est approuvée le 22 mars 1862. D’une longueur totale de $490 \mathrm{~m}$ depuis la cour de la gare jusqu'à l'axe de la route impériale $\mathrm{n}^{\circ} 12$, elle se compose de deux parties. La première, parallèle à l'axe du chemin de fer, a une largeur totale de 25,50 $\mathrm{m}$ dont $12 \mathrm{~m}$ de chaussée, $2 \mathrm{~m}$ de caniveaux pavés, et $11,50 \mathrm{~m}$ de trottoirs. En raison des impératifs militaires, sa surface est arasée afin de dégager entièrement l'environnement de la gare. La seconde, perpendiculaire à la porte de Landerneau, a une largeur de chaussée de 9,60 $\mathrm{m}$ et $2 \mathrm{~m}$ de caniveaux pavés. Le trottoir gauche est large de $8 \mathrm{~m}$ dans toute la longueur, tandis que la largeur du trottoir de droite varie de $8 \mathrm{~m}$ à $3,20 \mathrm{~m}$ à la hauteur de la place du Roi de Rome, la largeur totale de la voie s'établissant ainsi entre $24,40 \mathrm{~m}$ et $27,60 \mathrm{~m}$. Si cette artère présente une commodité pour le déplacement des voyageurs, elle a néanmoins l'inconvénient de leur imposer un détour ${ }^{14}$. En complément, un autre boulevard est également dessiné le long des fortifications, depuis la place du Château jusqu'à la place du Roi de Rome, reliant ainsi le port de commerce à la gare (fig. 2).

\section{La valorisation du paramètre stratégique du port et de I'arsenal}

À défaut d'un véritable aménagement esthétique de la ville de Brest, le chemin de fer devient plutôt synonyme d'un aménagement technique à travers la valorisation du port de commerce en construction et le paramètre stratégique de l'arsenal maritime. Il est prévu de desservir ces deux ensembles par un embranchement ferré à voie unique qui, techniquement, commence au lieu-dit Le Rody, situé sur la ligne principale, puis franchit le contrefort situé entre le Moulin blanc et Saint-Marc, pour suivre ensuite le bord de la rade jusqu'au port et enfin se diriger vers l'arsenal en passant sous les fortifications du château ${ }^{15}$. Le Conseil cénéral des Ponts et Chaussées approuve l'avant-projet présenté le 16 février 1861, mais limite cette approbation à la partie du tracé comprise entre le point de bifurcation de la ligne principale et le prolongement de l'axe du quai est du bassin à flot projeté. Ce programme, d'une longueur de $2854 \mathrm{~m}$, est ensuite définitivement approuvé

14- L'avenue est remise à la ville le 25 janvier 1865, alors que, parallèlement, la reconnaissance finale des travaux de terrassements et ouvrages d'art de la gare intervient le 4 avril 1865.

15- Planchat, le 17 juin 1860, suggéra de remplacer la voie unique originale par une double voie, convaincu qu'on en reconnaitrait la nécessité ultérieurement. Il fallut finalement attendre l'entre-deux-guerres pour voir le doublement de la voie du Rody enfin réalisé. 
par la décision ministérielle du 2 mars 1863. Une plate-forme de $630 \mathrm{~m}$ de longueur sur $35 \mathrm{~m}$ de largeur est aménagée au Rody, pour y disposer des voies de classement. Les terrassements et les travaux jusqu'au port, évalués à $750000 \mathrm{~F}$, sont confiés à l'entreprise Parris, chargée également du lot correspondant de la ligne Rennes-Brest. Une nouvelle enquête est organisée du 19 au 27 avril 1863, validant ainsi les dispositions initiales.

La question de l'embranchement militaire, alors ajournée pour des raisons techniques, est à nouveau abordée par le comité des fortifications le 29 janvier 1862. Jusqu'alors, le ministère de la Marine s'était en effet montré peu convaincu de sa nécessité et il faudra une observation appuyée du préfet maritime de Brest pour infléchir sa position ; la batellerie, alors prédominante dans les activités de transport, risquait en effet de devenir difficile à cause de la force du ressac contre les remblais du port. À l'arrivée, le projet de la Marine se révèle somme toute identique à celui des ingénieurs du chemin de fer présenté en 1861. Ainsi, en quittant le port, la voie ferrée entre en tunnel sous le bastion 19 bis, par une courbe de $300 \mathrm{~m}$ de rayon. Le souterrain, d'une longueur de $138 \mathrm{~m}$ pour $9 \mathrm{~m}$ de largeur, vient déboucher à l'aplomb du ravelin 15 du château, dans les fossés, lesquels sont approfondis de 12 à $14 \mathrm{~m}$. La voie franchit ensuite, en tranchée, le terre-plein de l'extrémité ouest de la place du château, puis aboutit sur le quai Tourville, à l'intérieur de l'arsenal. Le profil transversal est établi pour deux voies, mais dans l'immédiat une seulement est construite, tandis que l'autre est réservée provisoirement à la circulation des piétons et des voitures.

Le 7 juillet 1862, la commission mixte des Travaux publics admet l'exécution de l'ouvrage. L'entrée dans le mur d'escarpe de l'ouvrage 19 bis est organisée conformément aux dispositions proposées par le Génie, avec un système de deux baies séparées par un piédroit intermédiaire, de manière à pouvoir établir deux pont-levis, nécessaires pour assurer la fermeture de l'arsenal ${ }^{16}$. Le parc de l'artillerie de terre est réorganisé et la salle d'artifices est reconstruite dans les ouvrages 16 et 17 du château. Les travaux sont exécutés, après entente, par la Marine, excepté les ouvrages de l'entrée est du tunnel, le dressement des talus dans les tranchées du fossé de la fortification, et les nouveaux aménagements de l'artillerie de terre, lesquels sont réalisés par le Génie. La dépense totale se porte à $197000 \mathrm{~F}$, à savoir $146000 \mathrm{~F}$ pour la porte du tunnel

16- Les largeurs du pilier central et des portes sont respectivement fixées à 1,60 $\mathrm{m}$ et à $4,50 \mathrm{~m}$. 
et $51000 \mathrm{~F}$ pour la modification des ouvrages de la fortification ${ }^{17}$. La réalisation de cette voie marque la fin des travaux ferroviaires de la ligne Rennes-Brest, dont le montant global s'élève à 26 millions et demi de francs, non compris les dépenses à la charge de la Compagnie de l'Ouest (ballast, voie, édifices des stations). Le $1^{\text {er }}$ octobre 1865 , les quais de la Penfeld sont définitivement évacués et, le 2 octobre, le nouveau port entre en activité, profitant en outre de l'établissement officiel, depuis le 2 juin, de l'escale postale. Il est prévu, dans un avenir proche, de compléter l'ensemble par une gare maritime exclusivement réservée aux marchandises, à l'entrée est du site (fig. 2).

\section{Le consensus social de l'inauguration}

Brest, par un temps magnifique, est la dernière grande ville bretonne à être reliée au chemin de fer le mardi 26 avril $1865^{18}$. Pour célébrer l'événement, qui doit consacrer l'Empire aménageur du territoire, la municipalité a voté un crédit spécial de $80000 \mathrm{~F}$. La plupart des maisons et des édifices a été pavoisée du drapeau tricolore, tandis que, dans la rade et le port, les navires ont arboré leurs pavois. Dans cette atmosphère de liesse, tous les regards sont naturellement tournés vers le bâtiment des voyageurs où 900 invités ont pris place pour accueillir le ministre des Travaux publics, Béhic, attendu en fin d'après-midi ${ }^{19}$, mais aussi pour s'imprégner de l'ambiance du nouvel équipement. Celui-ci, selon les instructions du Génie, est majoritairement édifié en briques et en bois, matériaux qui facilitent son rapide sabotage en cas de danger militaire venant des terres. Malgré ces contraintes structurelles, la Compagnie de l'Ouest a conçu un ensemble soigné, condition nécessaire étant donné son caractère de gare terminus. Le plan du bâtiment épouse un développement en U tronqué, composé de deux ailes, le corps transversal étant pour sa part cantonné de deux élégants pavillons d'angle.

17- La porte du tunnel est remise à la Marine le 11 juillet 1868 . L'article 5 du procèsverbal de remise prévoit notamment, en temps de guerre, son placement sous l'autorité du commandant de la place (Service historique de la Défense, Armée de terre, A6 Brest/C 149).

18- En Bretagne, le chemin de fer arrive à Nantes en 1851, à Rennes en 1857, à Vannes, Lorient, Quimper et Saint-Brieuc en 1863, et à Napoléonville (Pontivy) en 1864. Les trajets vers Paris sont ainsi considérablement raccourcis, à l'exemple de Brest qui ne se trouve plus qu'à 16 heures de la capitale alors qu'auparavant le trajet en diligence s'effectuait en 62 heures.

19- 1000 autres places sont accordées dans l'enceinte extérieure de la gare aux personnes munies de billets spéciaux. Les officiers et fonctionnaires en uniforme y sont également admis. 
La façade principale suggère l'idée d'un dynamisme formel par la justesse de ses proportions et la présence d'un porche d'entrée central percé d'une grande baie en plein cintre, élancée sur deux niveaux. La halle métallique, avec sa grande verrière et le traditionnel lanternon, contribue à en renforcer l'ampleur en se détachant nettement à l'arrière-plan (fig. 3). Pour la presse, le bâtiment fait l'unanimité. En comparaison avec d'autres gares importantes, la distribution de ses pièces « est bien entendue » et il « semble d'une capacité suffisante » pour les besoins du moment $\mathrm{t}^{20}$.

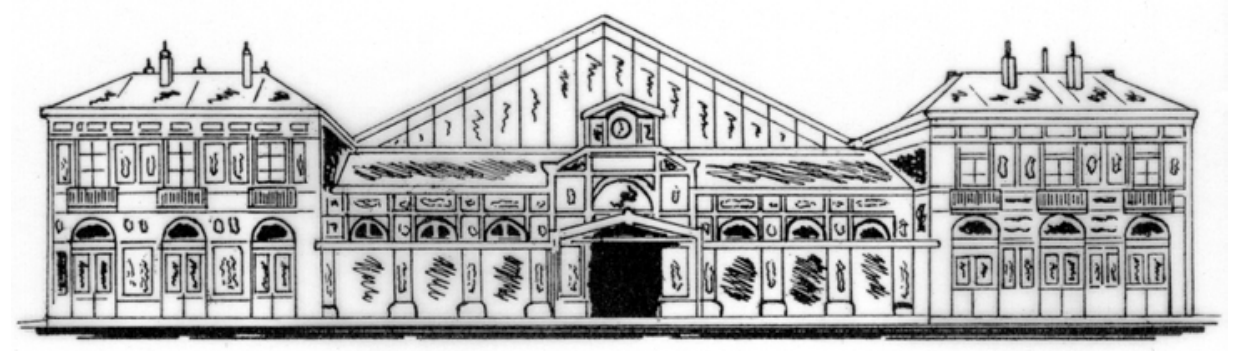

Figure 3. Le bâtiment des voyageurs de Brest en 1865. Dessin C. Le Bollan.

La cérémonie débute à 15 h 30, moment choisi par le clergé pour investir processionnellement la gare. Peu après, aux sons de la musique de la flotte, un cortège, avec à sa tête le préfet maritime, pénètre à son tour dans l'enceinte. À $17 \mathrm{~h}$, le train officiel, mené symboliquement par la locomotive Le Finistère, entre en gare et le ministre, accompagné de diverses personnalités, est chaleureusement accueilli. L'archiprêtre Mercier, de la paroisse de Saint-Martin, prend alors la parole, et dans un discours "pastoral et progressif, llui présente] les vœux du clergé breton » pour Napoléon III, « [invoque], avec patriotisme, sur les voies ferrées finistériennes les bénédictions de Dieu, et [rappelle] les efforts du gouvernement, les travaux intelligents des ingénieurs, le concours de l'autorité administrative, le dévouement éclairé de la municipalité pour que [le] pays [prenne], à l'aide du chemin de fer et du port de commerce, la position considérable que lui assigne son

20- L'Armoricain, 13 avril 1865. Le site comprend par ailleurs une remise à machines, une halle à marchandises et des ateliers divers. 
importance topographique ». Le ministre prononce ensuite une courte allocution par laquelle il fait connaitre « la satisfaction de l'Empereur de la transformation de Brest, et du vif désir de Sa Majesté de compléter l'œuvre de régénération de [la] cité, réalisant ainsi la grande pensée nationale de Napoléon Premier ». Puis, avant de se rendre à la réception officielle, il décore les ingénieurs en chef Planchat et Fessard ${ }^{21}$ de la croix d'officier de la Légion d'Honneur.

Durant le dîner, le maire ne manque pas de porter un toast à l'Empereur. Puis le ministre conclut par un dernier discours dans lequel il affirme solennellement « qu'en ce jour particulier [...] la Bretagne sort de son isolement ; les deux grandes lignes qui l'enlacent, reliées l'une à l'autre par plusieurs voies secondaires, forment un réseau à mailles étroites appelé à se resserrer encore et qui ne saurait laisser échapper aucun élément de richesse et de fécondité. Cette intéressante contrée n'en est plus réduite à vivre pour ainsi dire sur elle-même ; son activité propre devenue solidaire de l'activité générale du pays, se confond désormais avec elle [...]. La France est la bonne mère de famille qui aime à rapprocher et à serrer autour d'elle tous ses enfants ${ }^{22}$. Ces propos mettent, d'une certaine manière, un terme définitif aux querelles intestines qui ont divisé les Bretons pour la définition du réseau ferré régional ${ }^{23}$. L'heure est au consensus, l'aménagement du territoire sous le Second Empire demeurant véritablement l'affaire de tous.

Au vu des enseignements qui viennent d'être présentés, le projet ferroviaire brestois se révèle finalement symptomatique des relations délicates qui opposent les militaires aux instances civiles en matière de travaux publics ${ }^{24}$. En l'espace de vingt-trois années, de 1842 à 1865, l'armée a ainsi réussi à s'approprier en quelque sorte le projet local en l'exploitant habilement pour ses installations. L'orientation esthétique de la gare, dans sa connotation urbaine, s'est progressivement effacée devant la nécessité stratégique et les chances d'incorporer la gare à l'intérieur de l'intra-muros ou sur les terre-pleins portuaires n'étaient que

21- Charles-Jules Fessard (1815-1879) est engagé par la Compagnie de l'Ouest le

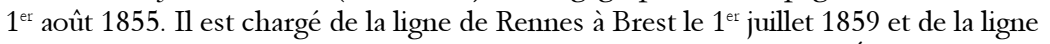
de Napoléonville à Saint-Brieuc le 10 juillet 1861 (Fichier Richard, École nationale des Ponts et Chaussées).

22- L'Armoricain, 27 avril 1865.

23- Le débat sur le réseau breton va en effet diviser les partisans de lignes littorales, finalement retenues, et les partisans d'une ligne centrale.

24- Les relations étaient d'autant plus délicates que sept acteurs étaient au total sollicités (le Génie, le domaine maritime, l'artillerie, la marine, les Ponts et Chaussées, la Compagnie des chemins de fer de l'Ouest et la municipalité). 
pures illusions. Cette dernière option, pourtant, aurait pu être la plus cohérente en raison de la vocation maritime de Brest et des espoirs de développement économique placés dans le nouveau port de commerce. L'espace ne manquait pas et techniquement tout semblait possible. Mais un examen plus approfondi des lieux révèle que tout ne plaidait pas forcément dans ce sens. Le port était en effet situé en contrebas de la ville et l'importante dénivellation, si la gare avait été implantée en cet endroit, aurait été source de fatigue pour les voyageurs et d'une image peu flatteuse de la cité à l'arrivée des trains. Un adoucissement de la topographie était toujours possible mais cette éventualité semble aujourd'hui peu plausible. La construction de la ligne de l'Ouest promouvait tout d'abord un principe d'économie et, pour les décideurs de l'époque, une telle dépense n'était pas prioritaire. Mais c'était surtout compter avec un vieux réflexe conservateur qui, inconsciemment, avait imprégné la mentalité brestoise depuis deux siècles. Si la Grande Muette autorisa dès 1870 le percement de la porte Foy à l'emplacement du front 22-24, face à la gare, pour offrir à cette dernière une liaison directe avec la ville, il en alla autrement de l'arsenal maritime, le « cœur » de Brest, qu'il convenait de protéger à outrance. Dans cette optique, le cours d'Ajot, promenade élevée qui dominait le port au sud de la ville, représentait un obstacle conséquent permettant de dissuader tout assaillant ; pour cette raison, tout adoucissement de cette pente était proscrit. L'armée cherchait avant tout à défendre ses prérogatives et elle n'était nullement disposée à tolérer la proche présence d’édifices ferroviaires qui, subsidiairement, auraient pu devenir de sérieux points d'appui pour un ennemi audacieux. Une telle vision des choses avait-elle réellement un sens alors que la navigation à vapeur et les progrès de l'artillerie prenaient toute leur place dans les nouveaux systèmes stratégiques? La question reste posée, tout comme celle de savoir si une gare maritime concentrant à la fois des prestations pour les voyageurs et les marchandises aurait véritablement contribué à davantage valoriser le port. Car celui-ci présenta rapidement un bilan négatif, causé en particulier par une mauvaise organisation. La gare maritime vouée aux marchandises selon le projet de 1865 se résuma en fait à quelques voies de quais... démunies de tout bâtiment destiné à l'enregistrement du trafic de petite vitesse. Les usagers étaient ainsi contraints d'effectuer des allers et venues, via le boulevard extérieur, avec celui qui existait près du bâtiment des voyageurs! D’autres facteurs aggravèrent la situation comme la position ambiguë de la Compagnie de l'Ouest par l'application de tarifs élevés continuellement remis en cause par la chambre de commerce de Brest ou encore les règlements contraignants. La sanction fut immédiate et conduisit, dès 1874, à la suppression de l'escale transatlantique, 
mettant ainsi un terme cinglant à l'enthousiasme né du Second Empire. Ce pathétique coup d'arrêt aura pour conséquence d'intensifier le retard du port de Brest sur ses concurrents et il faudra attendre le décret présidentiel du... 29 décembre 1894 pour voir enfin la Compagnie de l'Ouest obtenir la concession des voies ferrées portuaires! Pour autant, le projet technique était une nouvelle fois orphelin de tout faisceau de triage et de bâtiment représentatif de la compagnie. Ironie de l'histoire, c'est un autre type de relation transatlantique qui permit au port de se métamorphoser. Le site fut en effet retenu pour accueillir une partie du corps expéditionnaire américain en 1917, événement qui eut pour principal avantage de renforcer son potentiel technique par l'apport de grues à fort tonnage, de voies supplémentaires et de faisceaux de triage. Le Réseau des chemins de fer de l'État, qui remplaça la Compagnie de l'Ouest à partir de 1909, bénéficia ainsi d'un substrat plus favorable qu'il sut agrémenter, durant l'entre-deux-guerres, d'un petit bâtiment d'exploitation. La même période vit également la concrétisation tant attendue de l'extension urbaine de Brest, suite au déclassement des fortifications. Comme un juste retour des choses, la gare de voyageurs sera l'un des supports de ce projet ; reconstruite de 1932 à 1936 par l'architecte Urbain Cassan, elle marquera ainsi symboliquement, par sa tour-horloge en béton armé, la transition parfaite entre tradition et modernité. 